\title{
Boole-WebLab-FPGA: Creating an Integrated Digital Electronics Learning Workflow Through a Hybrid Laboratory and an Educational Electronics Design Tool
}

\author{
http://dx.doi.org/10.3991/ijoe.v9iS8.3362 \\ Javier García-Zubía, Ignacio Angulo, Luis Rodriguez-Gil, Pablo Orduna, Olga Dziabenko, Maria Güenaga \\ University of Deusto, Bilbao, Spain
}

\begin{abstract}
During the last years the presence of remote laboratories has been growing. However, many labs focus on providing a very specific experience, intended to prove a specific point or to carry out a specific practice, rather than to help the student acquire a wider range of skills through several different uses of the lab. This paper describes the creation of Boole-WebLab-FPGA, a system which integrates Boole-Deusto -an educational electronics design tool- and WebLab-Deusto-FPGA. The latter is a hybrid remote laboratory which lets users program a real FPGA board remotely. This real, physical board has been given the capability to interact with not only real hardware (LEDs), but also with virtual simulations of more complicated models, such as a watertank. The integration of these tools in a single system provides an attractive workflow for teachers and students. Students can design a combinational circuit easily through Boole-Deusto and immediately (in the same lecture session, if they so wish) try it on real hardware. Because additionally that real hardware can also be interacting with a virtual simulation, a wide range of experiments can be proposed and the replay potential of the laboratory is increased.
\end{abstract}

Index Terms-remote laboratories, digital electronics, hybrid laboratories, augmented reality, virtual laboratories

\section{INTRODUCTION}

In an engineering degree learning how to design and implement a logical system is a key part of the syllabus. The design process starts in the classroom through traditional means (only in some cases with computer aid) and the implementation is made in a lab with real instruments and devices. The laboratory is of utmost importance in the teaching and learning process [1].

In the field of digital electronics it is very common to teach using software tools for the analysis and design stage, and then laboratories to implement and test the designed circuits. But from the point of view of teachers and students, not all approaches to this are equally effective.

Boole-WebLab-FPGA, presented in this paper, is the combination of two systems. The first, Boole-Deusto, is an educational software that allows students to design a digital circuit step by step. The second, WebLab-FPGA, is a remote laboratory to implement the previously designed system on a real FPGA board. WebLab-FPGA also offers a hybrid laboratory, in which the real, physical board that the student programs can interact not only with the student and with real hardware, but also with a potentially complex virtual model, such as a Watertank. In the following sections this layer will be explained in greater detail, and an example of a laboratory (a virtual Watertank, with two water pumps and water level sensors, to be controlled by the real FPGA) will be described.

\section{BOOLE-DEUSTO}

The Boole-Deusto [2] software tool was developed and released for the first time around year 2000 [3]. Its main objective was to help teachers and students in the teaching and learning process of digital electronics, especially for introductory courses. Boole-Deusto focuses only on bitlevel systems. It transforms the system step by step from the original problem statement (proposed to the students) to the logical circuit: statement, truth table (combinational system) or finite state machine (sequential system), K maps, Boolean expression and digital circuit.

For example: the level of a water tank is controlled by two pumps (P1 and P2) depending on the values of three sensors -HL: high level of water, ML: medium level of water, LL: low level of water-. The Fig. 1 shows the truth table of the system, the K-map and the logical circuit.
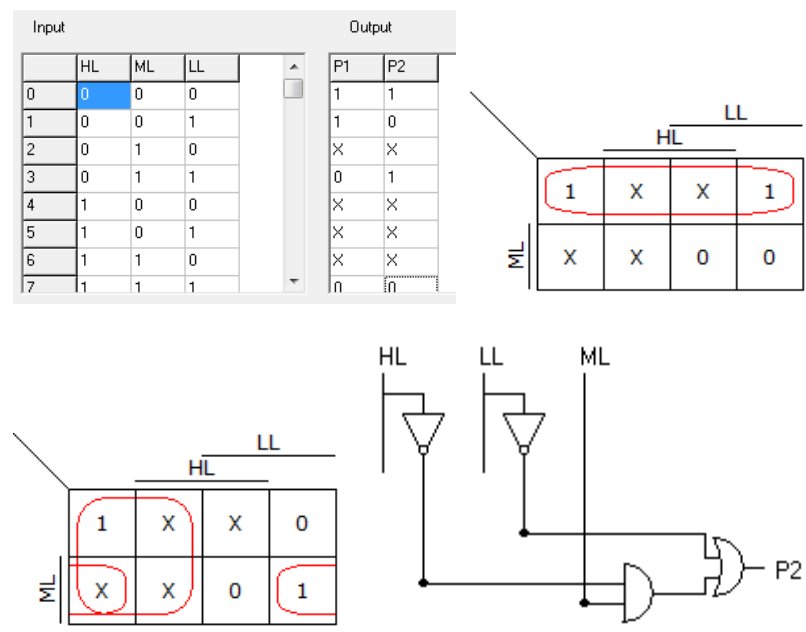

Figure 1. Defining the logic for controlling two pumpse with BooleDeusto. 
In general, professional software tools such as Proteus, LogicSim, etc. do not accompany the teachers and students through all the steps because their focus is only in the result: the digital circuit. Through Boole-Deusto, however, users can be taken through the whole process step by step, providing them with full control, allowing them to modify the system at any stage.

\section{CONnECting Boole-Deusto to WebLaB- DEUSTO}

Once users finish the design process, if they click on the WebLab-Deusto button a browser opens and they can directly access a FPGA controlled by WebLab-Deusto.

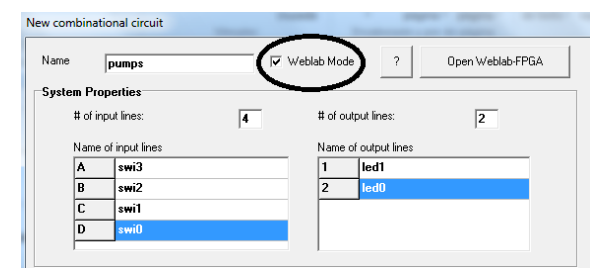

Figure 2. WebLab Mode

When this is done, a browser will be opened. Once the VHDL file that Boole-Deusto generates has been uploaded, the code is automatically synthesized and programmed into the remote FPGA board. Once this process has finished, users will not only be able to see how the system runs, but interact with it through virtual switches and buttons, as well. For instance, if the tank is not completely full of water (011 in switches sw2, sw1 and sw0) the pump P2 will be ON (led 1).
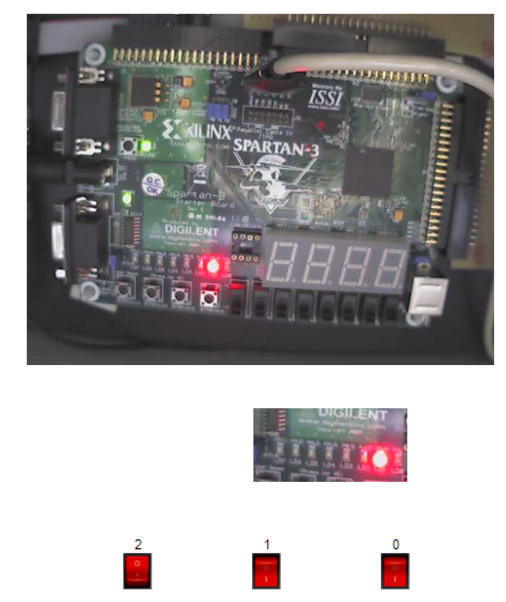

Figure 3. User's system running on a real, remote FPGA

As Fig. 3 shows, users can interact with the board, which has LEDs as outputs. Through them, they can check whether their system behaves as expected. The LEDs most of the time simulate other, more complex outputs, such as the Watertank that has been described throughout this example. Though in many cases this is appropriate, at it certainly makes many different exercises possible, at times students may find them somewhat similar and not very engaging. To solve that issue and to be able to provide whole new sets of exercises and different experiences, the next step involves improving the user experience and increasing the educational potential of the experiments through augmented reality. This new optional layer is described in more detail in the next section of this work.

\section{WEBLAB-FPGA-WATERTANK}

The standard WebLab-FPGA experiment is the one that has been described so far throughout this work. It is a traditional remote laboratory. Remote users submit their files, they are synthesized and programmed in the server, and the results can be seen and acted upon through a Webcam.

Though in many circumstances this is very useful and exactly what is wanted of a remote lab, this approach has certain limitations. The remote FPGA boards, which are pre-made and expensive, only have a limited set of outputs: several red LEDs. As a result, teachers and students may perceive that the variety of possible exercises is limited. They can certainly create an unlimited number of different exercises, but they may all be perceived as similar in nature. This can lower the students' engagement level and decrease their willingness to successfully complete the proposed exercises and to learn.

WebLab-FPGA-Watertank was created to tackle this problem. Built upon the standard WebLab-FPGA, it adds a virtual layer, making it a hybrid laboratory. That is, a laboratory which has both virtual and real remote components. Most hybrid labs do not make use of both components at the same time. Instead, the typical hybrid lab first offers a simulation through its virtual layer, and only then executes the experiment on real hardware. However, WebLab-FPGA-Watertank's nature is very different. The real and virtual layers interact with each other in real-time, in both directions, and one does not make sense without the other.

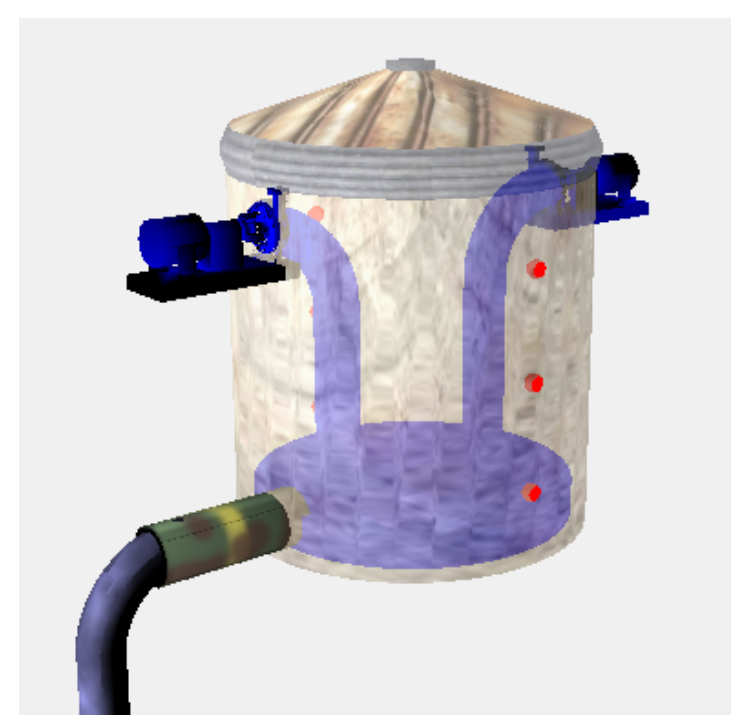

Figure 4. Virtual watertank model, with red spheres depicting level sensors and the two water pumps active. The model is to be controlled by the real FPGA board.

The main targets of the WebLab-FPGA-Watertank laboratory were to maintain maximum realism. Therefore, the controller (the FPGA board) had to be absolutely real, and not virtual. At the same time, a complex environment to control with the FPGA was required, so that engaging and complex exercises could be proposed. To achieve this, WebLab-FPGA-Watertank adds a virtual environment on which the real FPGA board can act. Hence, the virtual environment is essentially a new set of very complex inputs and outputs for the real board. Fig 4. shows exactly the characteristics of this model. It has three level-sensors 
available (represented as three small red spheres), two water pumps (close to the top) and a pipe with random water demand (close to the bottom).

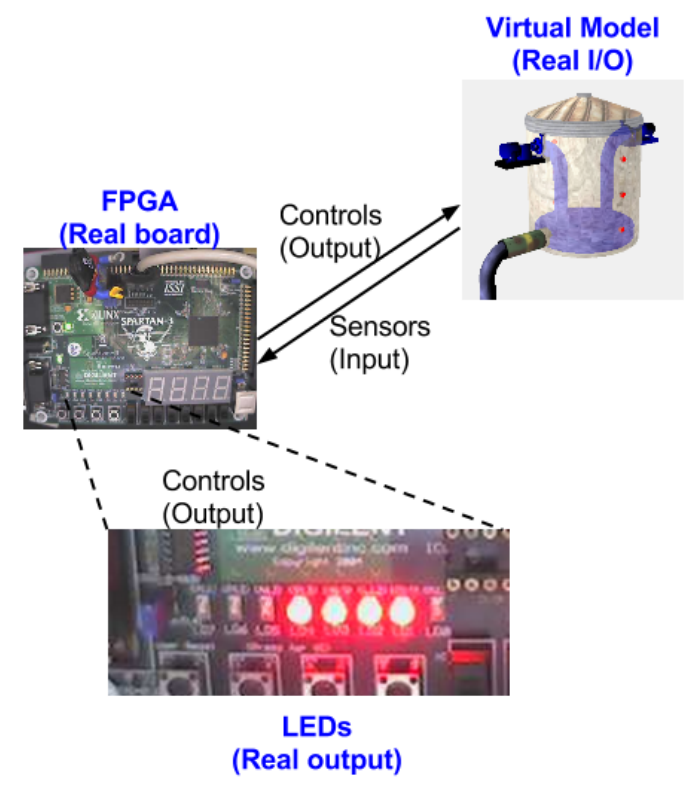

Figure 5. Weblab-FPGA-Watertank scheme. The FPGA board interacts with the virtual model in both directions. It has the water pumps as outputs and the water-level sensors as inputs.

The key here, however, is that these virtual inputs and outputs interact with the real FPGA board. The program that users specified on the standard (non-hybrid) FPGA experiment would have switches as inputs and LEDs as outputs. Now, the switches are actually the sensors from the virtual model (the water-level sensors) and the LEDs are the water pumps. Thus, if the user's program, for instance, turns the first led on, it is actually turning on the water pump in the virtual model as well. This is depicted in Fig 5.

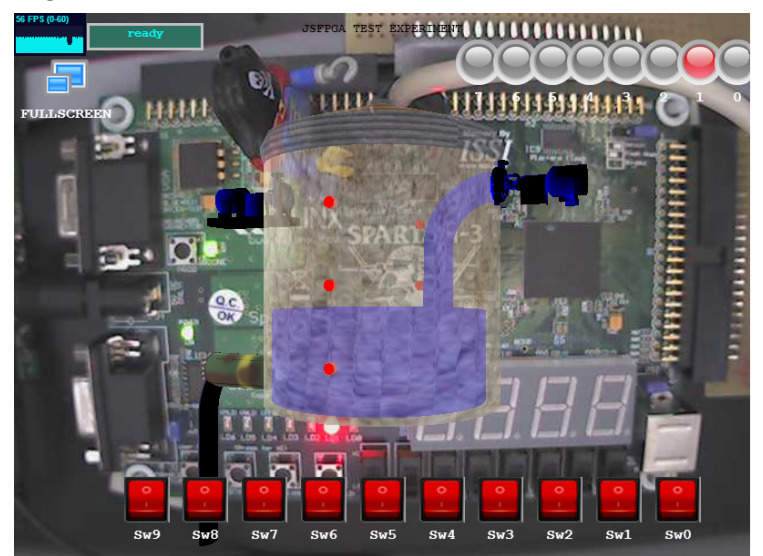

Figure 6. WebLab-Deusto-FPGA. The remote user's program has turned the second LED on and hence has also turned on one of the two virtual water pumps.

Fig 6. depicts the actual FPGA-Watertank experiment as seen by end-users. The virtual model is displayed on top of the FPGA board. The FPGA board image is streamed from the webcam and is the same board that is actually controlling both the real LEDs and the virtual watertank model. The technology that powers this client is
WebGL [5][4] and is compatible (or will be soon) with most browsers.

The model has three level-sensors available (represented as small red spheres), two water pumps, and an output (whose flow varies randomly, representing water demand). The aim of the VHDL code to program in the FPGA would be to keep the water level of the deposit between a certain threshold, never overflowing and never going too low. Technically, the virtual model takes the LEDs as inputs, which lets users control the water pumps easily through VHDL code. The virtual sensor inputs are also readily available to the VHDL code, replacing some of the switches that can normally be controlled manually.

\section{CONCLUSIONS AND FUTURE WORK}

After the design process, students can either print a diagram of the circuit or obtain the VHDL program which describes it. Then, they should move to a laboratory to implement that circuit and analyze if it runs properly or not. Generally, as stated, the design is made in a standard classroom or at home, whereas the implementation is made in an electronics laboratory with specialized equipment. The aim of this new approach has been to connect Boole-Deusto with a remote laboratory (WebLab-Deusto), in order for students to be able to remotely test their designs. This is often easy, straightforward and convenient, because no wiring or specialized equipment is required. An internet connection is enough to get the designed system implemented in a real, physical board, and to interact with it and check its response. The Boole-Deusto program does all the work, the students do not wire their circuits nor write VHDL code.

The system has been designed and implemented in such a way that it is relatively easy to add new virtual models on top of the real FPGA. Therefore, in the future, new models will probably be implemented, so that an even wider variety of experiments and experiences can be offered to the students. Examples of some of the virtual models that have been considered are a factory qualitytesting simulation, and the controls of a hypothetical (and simplified) hydroelectric facility.

\section{ACKNOWLEDGEMENT}

WebLab-Boole-Deusto is part of the OLAREX project to promote and to facilitate science and technology among young people in secondary schools. No. 518987-LLP-12011-1-ES-KA3-KA3MP funded with support from the Lifelong Learning Programme (KA3 - ICT) from European Union

\section{REFERENCES}

[1] Feisel, L.; Rosa, A. (2005) "The Role of the Laboratory in Undergraduate Engineering Education", Journal Engineering Education, January 2005, pp: $121-130$.

[2] http://paginaspersonales.deusto.es/zubia

[3] Garcia-Zubia, J. "Educational software for digital electronics: Boole-Deusto", Proc. of IEEE Int. Conf. on MSE, pp: $20-22$, 2003.

[4] Leung, C., and Salga, A. "Enabling webgl". In Proceedings of the 19 th international conference on World Wide Web (pp. 13691370). ACM. April, 2010. http://dx.doi.org/10.1145/1772 $\underline{690.1772933}$

[5] Marrin, C. "Webgl specification". Khronos WebGL Working Group, 2011. 


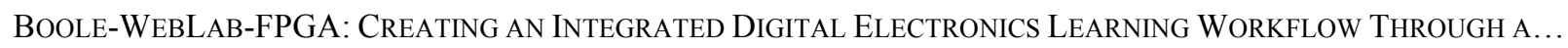

\section{AUTHORS}

Javier García-Zubía and Ignacio Angulo are with the Faculty of Engineering, University of Deusto, Bilbao, Spain, zubia@deusto.es

Luis Rodriguez-Gil, Pablo Orduna, Olga Dziabenko, and Maria Güenaga are with the Deusto Institute of Technology - DeustoTech, University of Deusto, Bilbao, Spain, luis.rodriguezgil@deusto.es.

This article is an extended and modified version of a paper presented at the International Conference exp.at'13, held 18-20 September 2013, in Coimbra, Portugal. Submitted 18 November 2013. Published as resubmitted by the authors 04 December 2013. 